\title{
NETosis and NADPH oxidase: at the intersection of host defense, inflammation, and injury
}

\section{Nikolaos G. Almyroudis 1,2, Melissa J. Grimm² ${ }^{2}$ Bruce A. Davidson ${ }^{3,4,5}$, Marc Röhm ${ }^{6}$, Constantin F. Urban ${ }^{6}$ and Brahm H. Segal ${ }^{1,2,7}$ *}

1 Division of Infectious Diseases, Department of Medicine, University at Buffalo School of Medicine, Buffalo, NY, USA

${ }^{2}$ Department of Medicine, Roswell Park Cancer Institute, Buffalo, NY, USA

${ }^{3}$ Department of Anesthesiology, University at Buffalo School of Medicine, Buffalo, NY, USA

${ }^{4}$ Department of Pathology and Anatomical Sciences, University at Buffalo School of Medicine, Buffalo, NY, USA

${ }^{5}$ Veterans Administration of Western New York Healthcare System, Buffalo, NY, USA

${ }^{6}$ Laboratory for Molecular Infection Medicine Sweden, Department of Clinical Microbiology, Umeå University, Umeå, Sweden

${ }^{7}$ Department of Immunology, Roswell Park Cancer Institute, Buffalo, NY, USA

\section{Edited by:}

Marko Radic, University of Tennessee, USA

\section{Reviewed by:}

Heather Parker, University of Otago, Christchurch, New Zealand

Jinfang Ma, Johns Hopkins University, USA

\section{*Correspondence:}

Brahm H. Segal, Division of Infectious Diseases, Department of Medicine,

Roswell Park Cancer Institute,

Elm and Carlton Streets,

Buffalo, NY 14263, USA.

e-mail: brahm.segal@roswellpark.org
Neutrophils are armed with both oxidant-dependent and -independent pathways for killing pathogens. Activation of the phagocyte nicotinamide adenine dinucleotide phosphate (NADPH) oxidase constitutes an emergency response to infectious threat and results in the generation of antimicrobial reactive oxidants. In addition, NADPH oxidase activation in neutrophils is linked to activation of granular proteases and generation of neutrophil extracellular traps (NETs). NETosis involves the release of nuclear and granular components that can target extracellular pathogens. NETosis is activated during microbial threat and in certain conditions mimicking sepsis, and can result in both augmented host defense and inflammatory injury. In contrast, apoptosis, the physiological form of neutrophil death, not only leads to non-inflammatory cell death but also contributes to alleviate inflammation. Although there are significant gaps in knowledge regarding the specific contribution of NETs to host defense, we speculate that the coordinated activation of NADPH oxidase and NETosis maximizes microbial killing. Work in engineered mice and limited patient experience point to varying susceptibility of bacterial and fungal pathogens to NADPH oxidase versus NET constituents. Since reactive oxidants and NET constituents can injure host tissue, it is important that these pathways be tightly regulated. Recent work supports a role for NETosis in both acute lung injury and in autoimmunity. Knowledge gained about mechanisms that modulate NETosis may lead to novel therapeutic approaches to limit inflammation-associated injury.

Keywords: NETs, NADPH oxidase, neutrophils, inflammation, injury

\section{INTRODUCTION}

Neutrophils are armed with a broad repertoire of tools for killing pathogens. Activation of the phagocyte nicotinamide adenine dinucleotide phosphate (NADPH) oxidase constitutes an emergency response to infectious threat and results in the generation of antimicrobial reactive oxidant intermediates (ROIs). In addition to oxidant-dependent host defense, neutrophils harbor proteases, antimicrobial peptides, lactoferrin, and other antimicrobial constituents that damage and kill microbes. Activation of NADPH oxidase in neutrophils is linked to activation of intracellular granular proteases and to generation of neutrophil extracellular traps (NETs). NETs are composed of an extracellular network of chromatin bound to granular and specific cytoplasmic proteins that can target extracellular pathogens.

NETosis can be induced in vitro by conditions that lead to robust NADPH oxidase activation [e.g., stimulation with phorbol myristate acetate (PMA)], and is triggered in vivo during states of emergency, such as infection and conditions mimicking sepsis, such as transfusion-associated acute lung injury (Caudrillier et al., 2012; Yipp et al., 2012). In this setting, NETosis in dead or dying neutrophils may amplify microbial killing. Whereas neutrophil apoptosis leads to non-inflammatory physiological cell death, NETosis results in the extracellular release of proteases and other injurious neutrophil constituents that can exacerbate inflammatory injury (Narasaraju et al., 2011; Caudrillier etal., 2012; Thomas et al., 2012).

We review the link between NETosis and NADPH oxidase activation and their effects on host defense and modulation of inflammation and injury. A greater understanding of these pathways may lead to novel therapeutic approaches to limit inflammation-associated injury.

\section{HOW NEUTROPHILS DIE}

One of the most important aspects of regulation of acute inflammation relates to its termination. Neutrophils recruited to sites of microbial invasion or tissue injury are activated by microbial products (e.g., endotoxin and formylated peptides), damage-associated molecular patterns (DAMPs; Zhang et al., 2010), and cytokines and chemokines within the inflammatory milieu. While this acute inflammatory response is critical for host defense, subsequent 
termination of neutrophilic inflammation and transition to inflammatory responses that mediate tissue repair (e.g., M2 or alternative macrophage polarization; Sica and Mantovani, 2012) are necessary to limit tissue injury.

Neutrophil homeostasis involves production and death of an extraordinarily large population of cells. The lifespan of circulating neutrophils has been estimated to be $<1$ day; however, a recent in vivo labeling study showed that the average estimated circulating human neutrophil lifespan was considerably longer (5.4 days; Pillay et al., 2010). During infection and other major stressors, granulopoiesis and circulating neutrophil counts can increase dramatically; these conditions also modulate neutrophil survival and death as well as the mode of death.

Apoptosis is the default mode of neutrophil death. Apoptosis is stimulated by a number of factors [e.g., members of the tumor necrosis factor (TNF) cytokine family], and is regulated by specific caspases and members of the Bcl-2 family of proteins (Croker et al., 2011; Geering et al., 2011). Factors that can delay neutrophil apoptosis include lipopolysaccharide (LPS), granulocyte colonystimulating factor (G-CSF), granulocyte-macrophage colonystimulating factor (GM-CSF), and proinflammatory cytokines (Coxon et al., 1999; Klein et al., 2001; Garlichs et al., 2004; Akagi et al., 2008; Jun etal., 2011). Neutrophils are likely committed to apoptotic death by their constitutive co-expression of cell-surface Fas and Fas ligand, an autocrine mechanism that is suppressed by proinflammatory cytokines (Liles et al., 1996). In mice, phagocytosis of apoptotic neutrophils by macrophages reduces IL-23 production by these cells and downstream IL-17A production by $\mathrm{T}$ cells, thereby limiting granulopoiesis (Stark et al., 2005).

NETosis and necrosis of neutrophils are induced by different stimuli and have morphologically distinct features. Following toxin-induced necrosis, nuclear lobes in neutrophils lose their hypersegmented structure, but the nuclear envelope and granules remain intact (Fuchs et al., 2007). Nuclear decondensation and breakdown of the nuclear and granular membranes are unique features of NETosis (Fuchs et al., 2007). NETs are demonstrated by immunofluorescence showing mixing of nuclear (e.g., DNA and histones) and granular constituents [e.g., neutrophil elastase (NE)] on the extracellular surface of neutrophils (Brinkmann et al., 2010).

How neutrophils die likely affects their clearance and crosssignaling to monocytes/macrophages. At sites of inflammation, neutrophils undergo spontaneous apoptosis (Savill et al., 1989). In addition, neutrophil apoptosis can be induced by macrophages releasing death receptor ligands, such as TNF- $\alpha$ and Fas ligand (Brown and Savill, 1999; Yamashita et al., 1999; Renshaw et al., 2000). Macrophages recognize and ingest apoptotic neutrophils (Savill etal., 1989). Phosphatidylserine products are externalized by neutrophils early during apoptosis and stimulate phagocytosis of neutrophils by macrophages (efferocytosis), thus promoting resolution of inflammation (Frasch et al., 2011). In contrast, NETotic neutrophils display phosphatidylserine only after plasma membrane rupture (Fuchs et al., 2007). In addition, release of primary neutrophil granular proteins can recruit circulating monocytes to the site of inflammation, stimulate macrophages to produce cytokines, and enhance the ability of macrophages to phagocytose bacteria (Soehnlein et al., 2008a, 2009a,b).

\section{GENERATION OF NETS}

Neutrophil extracellular trap generation was first described by Brinkmann etal. (2004), who showed that neutrophils release granule proteins and chromatin that co-mingle in extracellular filaments that bind to and kill bacteria and degrade virulence factors. NETosis progresses through stages that are distinct from apoptosis and necrosis. The nuclei of neutrophils transition from hypersegmented lobes to a round, decondensed morphology. Peptidylarginine deiminase 4 (PAD4) converts histone tail arginine residues to citrulline, leading to loss of positive charge and chromatin decondensation (Neeli et al., 2009; Wang et al., 2009; Li et al., 2010; Farley et al., 2012). Later, the nuclear envelope and the granule membranes break down, allowing mixing of these components that are subsequently released as extracellular structures (Fuchs et al., 2007). Whereas NETosis has been considered to be an event following neutrophil death and breakdown of membranes, it is also possible for viable neutrophils to produce NETs (Yousefi et al., 2009; Yipp et al., 2012). Yipp et al. (2012) showed that during experimental infection, anuclear neutrophils with intact membranes formed NETs and were capable of migration and phagocytosis.

NETosis can be triggered by exposure of neutrophils to PMA, IL-8, LPS, interferon-gamma (IFN- $\gamma$ ), bacteria and fungi and their products, and complement-mediated opsonization (Brinkmann et al., 2004; Martinelli et al., 2004; Fuchs et al., 2007; Yamada et al., 2011; Saitoh et al., 2012; Yipp et al., 2012). Activated platelets can induce NETosis in neutrophils during bacterial sepsis, thereby capturing microbes and promoting their clearance (Clark et al., 2007; Phillipson and Kubes, 2011; McDonald et al., 2012). The proportion of activated neutrophils undergoing NETosis, the rapidity of NET generation, and the dependence on specific signaling pathways vary based on the stimulus (Hakkim et al., 2011). The RafMEK-ERK pathway is an upstream activator of NADPH oxidase in neutrophils and is involved in NETosis (Hakkim et al., 2011). RafMEK-ERK also upregulates expression of Mcl-1, an anti-apoptotic protein, suggesting that Raf-MEK-ERK may inhibit apoptosis to allow for NETosis (Hakkim et al., 2011). Several other signaling pathways can modulate NETosis. Inhibition of autophagy prevents chromatin decondensation in neutrophils and abrogates NETosis (Remijsen et al., 2011). Mammalian target of rapamycin (mTOR) mediates LPS-stimulated NET formation by post-transcriptional control of expression of hypoxia-inducible factor-1 alpha (HIF$1 \alpha$; McInturff et al., 2012). IFN- $\gamma$ produced by neutrophils can stimulate NETosis through an autocrine/paracrine process (Yamada et al., 2011).

In addition, specific neutrophil components that are NET constituents are also required for NET generation. NE is required for NET generation in pneumonia in mice (Papayannopoulos etal., 2010). In this model, NE traffics from primary granules to the nucleus where, together with myeloperoxidase (MPO), it degrades histones and promotes chromatin decondensation. MPO is also located in neutrophil primary granules and converts hydrogen peroxide to hypohalous acid, which has potent antimicrobial properties. MPO deficiency in humans leads to 
failure to generate NETs following stimulation with PMA or Candida (Metzler et al., 2011).

Neutrophil extracellular traps are also regulated by inhibiting pathways. SerpinB1 is an inhibitor of the neutrophil serine proteases. SerpinB1-deficient mice develop increased inflammation, tissue injury, and mortality following bacterial and viral infection (Benarafa et al., 2007; Gong et al., 2011). SerpinB1 restricted NETosis through a pathway that involves translocation of SerpinB1 from the cytoplasm to the nucleus early during NETosis (Farley et al., 2012). Curiously, recombinant SerpinB1 also inhibited NETosis through mechanisms that are unclear (Farley et al., 2012). MUNC13-4, a member of the MUNC13 family of proteins, is involved in exocytosis of lytic granules in cytotoxic $\mathrm{T}$ lymphocytes, and its deficiency leads to familial hemophagocytic lymphohistiocytosis (Feldmann et al., 2003). In neutrophils, MUNC13-4 mediates ROI generation, exocytosis of primary granules, and phagolysosomal maturation, but also inhibits NETosis (Monfregola et al., 2012). Finally, serum endonuclease DNase1 promotes degradation of NETs (Hakkim et al., 2010). Pathways that inhibit NETosis may limit neutrophil-mediated injury.

\section{RELATIONSHIP BETWEEN NADPH OXIDASE AND NETosis}

The phagocyte NADPH oxidase is comprised of a membranebound cytochrome consisting of gp91 ${ }^{\text {phox }}$ (phagocyte oxidase) and $\mathrm{p} 22^{\text {phox }}$. Upon activation, the cytoplasmic subunits, $\mathrm{p} 47^{\text {phox }}$, p67phox , and $\mathrm{p} 40^{\text {phox }}$ and rac translocate to the cytochrome. $\mathrm{NADPH}$ is oxidized to $\mathrm{NADP}^{+}$, and electrons are transported down a reducing potential gradient that terminates when oxygen accepts an electron and is converted to superoxide anion. Neutrophil NADPH oxidase activation occurs in response to microbes and to stimuli that can mimic infectious threat, such as formylated peptides, opsonized particles, integrin-dependent adhesion (Mocsai et al., 2002; Graham et al., 2007), and to activation of specific pathogen recognition receptors, such as dectin-1, which recognizes fungal cell wall-associated beta-glucans (Gantner et al., 2003; Goodridge et al., 2011).

Chronic granulomatous disease (CGD), an inherited disorder of NADPH oxidase, is characterized by recurrent life-threatening bacterial and fungal infections. Patients with only residual NADPH oxidase activity in neutrophils have a better prognosis than those with completely absent oxidase function (Kuhns et al., 2010). CGD is also associated with severe inflammatory complications, such as Crohn's-like inflammatory bowel disease (Marciano et al., 2004; Segal et al., 2011).

Activation of NADPH oxidase in neutrophils is linked to the release of cationic proteins from an anionic proteoglycan matrix within primary granules (Reeves et al., 2002). In this model, the released neutrophil serine proteases become activated and can target phagocytized microbes. NADPH oxidase can also stimulate NETosis. PMA-stimulated NET generation requires NADPH oxidase, while MIP-2 induces NETosis independently of NADPH oxidase (Farley et al., 2012). In pneumococcal lung infection, NETosis of lung neutrophils was reduced, but not eliminated, in NADPH oxidase-deficient mice (Yamada et al., 2011). Neutrophils from CGD patients are defective in NETosis, and gene therapy results in restored NETosis in NADPH oxidase-competent neutrophils in vitro (Fuchs et al., 2007; Bianchi et al., 2009).

Neutrophil NADPH oxidase can also modulate apoptosis. NADPH oxidase stimulates phagocytosis-induced apoptosis (Coxon etal., 1996). TNF- $\alpha$ and Fas ligand can both induce apoptosis in neutrophils, but through distinct signaling pathways; NADPH oxidase was required for TNF- $\alpha$-stimulated, but not Fas ligand-stimulated, apoptosis (Geering et al., 2011). Accelerating neutrophil death and clearance are likely to be important modes by which NADPH oxidase limits acute inflammation. Potentially, the intensity or kinetics of ROI generation may modulate the balance between apoptosis versus NETosis.

\section{NADPH OXIDASE AND NETs IN HOST DEFENSE}

NADPH oxidase can potentially target pathogens through a multistep process: direct antimicrobial effect of ROIs; intracellular activation of proteases that can target phagocytized pathogens; and generation of NETs that can attack extracellular pathogens. NETs can mediate host defense by trapping microbes thereby limiting their spread and by exposing them to high concentrations of several antimicrobial products. NET constituents can target different pathogens. For example, NE can degrade certain virulence factors of pathogens (Belaaouaj et al., 2000; Weinrauch et al., 2002). Calprotectin is a NET constituent that mediates nutritional immunity by sequestering divalent metal ions and targets Candida and Aspergillus species (Urban et al., 2009; Bianchi et al., 2011). However, the contribution of NET generation to host defense in vivo is difficult to determine because there are no genetic defects that selectively disable NETosis while leaving all other immune pathways intact. Therefore, it remains elusive whether the major role of NETs is to trap versus directly kill pathogens in vivo. In addition, the biological activity of individual cellular components released into NETs is undetermined. With these gaps in knowledge, the host defense contribution of NETosis versus that achieved by intracellular killing by intact neutrophils remains unsettled (Nauseef, 2012).

Specific pathogens may target NETs or exploit NET products to enhance bacterial invasion. For example, nuclease expression by Staphylococcus aureus degrades NETs and augments pathogen virulence in vivo (Berends et al., 2010). In addition, bacteria may exploit NET products to enhance microbial virulence. Shigella flexneri binds to cationic granular proteins expressed in NETs, which enhances bacterial adherence to and invasion of epithelial cells (Eilers et al., 2010). Paradoxically, excessive NETosis may impair host defense in vivo. SerpinB1-deficient mice had increased lung neutrophil NET generation but defective bacterial clearance compared to wild type mice in Pseudomonas aeruginosa pneumonia (Farley et al., 2012). While SerpinB1 may be required for host defense independently of its role in NETosis, these results raise the potential for injury caused by excessive NETosis abrogating antibacterial killing.

Neutrophil elastase and MPO are located in neutrophil primary granules, are constituents of NETs, and are required for NET generation in response to specific stimuli (described above). Therefore, deficiencies in these pathways can provide some insight into the role of NET constituents in host defense. The neutrophil serine proteases, NE, cathepsin-G (CG), and proteinase 3 (PR3), 
are activated by lysosomal cysteine protease cathepsin C/dipeptidyl peptidase I (DPPI; Pham et al., 2004). Papillon-Lefèvre syndrome is a rare autosomal recessive disease resulting from loss-offunction mutations in the DPPI gene locus that is characterized by palmoplantar hyperkeratosis, periodontitis leading to loss of teeth, and severe bacterial infections, including liver abscesses (Almuneef et al., 2003; Pham et al., 2004). MPO-deficient mice have defective candidal killing in vivo (Brennan et al., 2001) and neutrophils from MPO-deficient patients are impaired in the ability to limit growth of extracellular Candida albicans (Metzler et al., 2011). However, MPO deficiency in humans is usually asymptomatic, although severe candidal infections have been observed in patients with co-existing diabetes (Cech et al., 1979a,b).

NADPH oxidase deficiency leads to a more severe phenotype than Papillon-Lefevre or MPO deficiency. For example, patients with CGD are at high risk for invasive aspergillosis and specific bacterial pathogens (e.g., Burkholderia cepacia, Serratia marcescens, and Nocardia species; Winkelstein et al., 2000; van den Berg et al., 2009). Consistent with these clinical observations, CGD mice were highly susceptible to infection by Aspergillus and by B. cepacia, while $\mathrm{NE}^{-/-} \times \mathrm{CG}^{-/-}$mice and DPPI-deficient mice were resistant (Vethanayagam et al., 2011).

Additional studies point to specific host defense functions of neutrophil proteases that are distinct from NADPH oxidase. Proteases in human neutrophils target Streptococcus pneumoniae (Standish and Weiser, 2009) and protease-deficient mice have increased susceptibility to pneumococcal pneumonia (Hahn et al., 2011). In contrast, neutrophil NADPH oxidase has variable effects on pneumococcal strains in vitro; CGD neutrophils have normal killing of pneumococci that produce peroxide, but defective killing of peroxide-deficient mutant strains (Pitt and Bernheimer, 1974; Shohet etal., 1974), suggesting that pathogen-derived reactive oxidants can complement the killing defect in CGD neutrophils.

Together, these observations in the clinic and in mouse models support NADPH oxidase, neutrophil serine proteases, and MPO having distinct functions in host defense. Interventions that deplete NETs in vivo (e.g., histone blocking antibody and DNase1; Caudrillier et al., 2012) may be useful to delineate specific host defense functions of NETs.

\section{NADPH OXIDASE AND NETS HAVE DISTINCT EFFECTS ON INFLAMMATION AND INJURY}

The generation of NETs can be a double-edged sword. On the one hand, they may promote pathogen killing. However, the same pathways that control microbial infection can also cause injury through a number of mechanisms. NET constituents can damage epithelial and endothelial cells, which can exacerbate inflammation-induced organ injury (Saffarzadeh et al., 2012). The interaction between NETs, platelets, and coagulation further illustrate the concept of the dual host defense and injurious potential of NETs. Platelet-derived defensins have intrinsic antimicrobial activities and can stimulate NETosis (Kraemer et al., 2011). NET constituents (NE, CG, and histones) can activate platelets and promote coagulation leading to intravascular thrombus growth that restrict tissue bacterial invasion (Fuchs et al., 2010, 2011; Massberg et al., 2010); however, these same mechanisms may promote an excessive coagulopathy and thrombosis resulting in endothelial cell injury and organ damage. Recent studies have shown that NETosis can drive transfusion-related acute lung injury and that depleting NETs was protective (Caudrillier et al., 2012; Thomas et al., 2012).

NETosis has also been implicated in the pathogenesis of autoimmune disorders, such as systemic lupus. Autoimmunity may be driven by a combination of factors related to aberrant NETosis, including direct damage to tissue, release of autoantigens that stimulate immune complexes, complement activation, and IFN- $\alpha$ release by plasmacytoid dendritic cells (Guiducci et al., 2010; Hakkim etal., 2010; Meyer-Hoffert and Wiedow, 2010; Garcia-Romo et al., 2011; Lande et al., 2011; Villanueva et al., 2011; Leffler et al., 2012). A subset of patients with systemic lupus have reduced ability to degrade NETs due to impaired serum DNase1 activity, and have a higher incidence of lupus nephritis (Hakkim et al., 2010).

While NETosis has, so far, been shown to enhance neutrophilmediated injury, the role of NADPH oxidase is more complex and likely involves interplay of injurious and protective pathways. NET constituents such as neutrophil proteases generally lead to augmented inflammation and tissue injury (Adkison et al., 2002; Hu and Pham, 2005; Raptis et al., 2005; Akk et al., 2008; Soehnlein etal., 2008b). However, studies in NADPH oxidase-deficient mice point to a more complex interaction between NADPH oxidase and acute lung injury that is contextdependent. NADPH oxidase worsened the severity of acute lung injury following influenza virus challenge in mice (Imai et al., 2008). While wild type and NADPH oxidase-deficient mice had similar levels of lung injury following LPS challenge (Sato etal., 2002), NADPH oxidase-deficient mice had greater lung neutrophil sequestration, but less lung injury compared to wild type mice in E. coli sepsis (Gao etal., 2002). In contrast, NADPH oxidase was protective in acid aspiration-induced lung injury. NADPH oxidase-deficient mice had increased airway neutrophilic inflammation and acute lung injury (measured as albumin leak), but less injury per recovered neutrophil, compared to wild type mice (Segal etal., 2007; Davidson et al., 2013). In addition, NADPH oxidase was required for optimal activation of Nrf2, an ROI-inducible transcriptional factor that stimulates cytoprotective and anti-inflammatory responses (Davidson et al., 2013). Thus, NADPH oxidase likely has a dual effect on inflammation-induced lung injury. While the immediate effects of NADPH oxidase activation leads to ROI generation and possibly NETosis that are predicted to be injurious, NADPH oxidase can also protect against injury by limiting neutrophilic inflammation and by inducing cytoprotective pathways, such as Nrf2.

\section{CONCLUSION}

In response to infectious threat, neutrophils go to war. NADPH oxidase is rapidly activated by specific microbial stimuli, leading to ROI generation. NADPH oxidase can activate neutrophil granular proteases within the phagolysosome, thereby targeting phagocytized pathogens. In addition, NADPH oxidase stimulates NETosis, a process that targets extracellular pathogens. While important for host defense, NETs are 
also implicated in the pathogenesis of a number of diseases, including acute organ injury and autoimmunity. Understanding mechanisms by which NADPH oxidase and its regulated pathways modulate inflammation and injury may identify novel therapeutic approaches.

There are several gaps in knowledge regarding NETosis. Although NETosis is induced by infection or conditions mimicking infectious threat, we do not have a good understanding of the molecular mechanisms that drive NETosis. NADPH oxidase can stimulate NETosis in certain settings and apoptosis in others; it is unclear how these dual effects are mediated. It is also unclear which pathways stimulate NETosis after neutrophil death and breakdown of membranes versus NETosis in living neutrophils. Additional questions relate to the relative contributions of NETosis versus intracellular killing of pathogens to host defense and whether the major function of NETs is to prevent spread of versus killing of pathogens.

\section{REFERENCES}

Adkison, A. M., Raptis, S. Z., Kelley, D. G., and Pham, C. T. (2002). Dipeptidyl peptidase I activates neutrophilderived serine proteases and regulates the development of acute experimental arthritis. J. Clin. Invest. 109, 363-371.

Akagi, T.,Saitoh, T., O’Kelly, J., Akira, S., Gombart, A. F., and Koeffler, H. P. (2008). Impaired response to GM-CSF and G-CSF, and enhanced apoptosis in C/EBPbetadeficient hematopoietic cells. Blood 111, 2999-3004.

Akk, A. M., Simmons, P. M., Chan, H. W., Agapov, E., Holtzman, M. J., Grayson, M. H., et al. (2008). Dipeptidyl peptidase I-dependent neutrophil recruitment modulates the inflammatory response to Sendai virus infection. J. Immunol. 180, 3535-3542.

Almuneef, M., Al Khenaizan, S., Al Ajaji, S., and Al-Anazi, A. (2003). Pyogenic liver abscess and PapillonLefèvre syndrome: not a rare association. Pediatrics 111, e85-e88.

Belaaouaj, A., Kim, K. S., and Shapiro, S. D. (2000). Degradation of outer membrane protein A in Escherichia coli killing by neutrophil elastase. Science 289, 1185-1188.

Benarafa, C., Priebe, G. P., and Remold-O'Donnell, E. (2007). The neutrophil serine protease inhibitor serpinb1 preserves lung defense functions in Pseudomonas aeruginosa infection. J. Exp. Med. 204, 19011909.

Berends, E. T., Horswill, A. R., Haste, N. M., Monestier, M., Nizet, V., and von Kockritz-Blickwede, M. (2010). Nuclease expression by Staphylococcus aureus facilitates escape from neutrophil extracellular traps. J. Innate Immun. 2, 576-586.
Bianchi, M., Hakkim, A., Brinkmann, V., Siler, U., Seger, R. A., Zychlinsky, A., et al. (2009). Restoration of NET formation by gene therapy in CGD controls aspergillosis. Blood 114, 2619-2622.

Bianchi, M., Niemiec, M. J., Siler, U., Urban, C. F., and Reichenbach, J. (2011). Restoration of antiAspergillus defense by neutrophil extracellular traps in human chronic granulomatous disease after gene therapy is calprotectin-dependent. J. Allergy Clin. Immunol. 127, 12431252.

Brennan, M. L., Anderson, M. M., Shih, D. M., Qu, X. D., Wang, X., Mehta, A. C., et al. (2001). Increased atherosclerosis in myeloperoxidasedeficient mice. J. Clin. Invest. 107, 419-430.

Brinkmann, V., Laube, B., Abu Abed, U., Goosmann, C., and Zychlinsky, A. (2010). Neutrophil extracellular traps: how to generate and visualize them. J. Vis. Exp. 36:e1724, doi: $10.3791 / 1724$

Brinkmann, V., Reichard, U., Goosmann, C., Fauler, B., Uhlemann, Y., Weiss, D. S., et al. (2004). Neutrophil extracellular traps kill bacteria. Science 303, 1532-1535.

Brown, S. B., and Savill, J. (1999). Phagocytosis triggers macrophage release of Fas ligand and induces apoptosis of bystander leukocytes. J. Immunol. 162, 480-485.

Caudrillier, A., Kessenbrock, K., Gilliss, B. M., Nguyen, J. X., Marques, M. B., Monestier, M., et al. (2012). Platelets induce neutrophil extracellular traps in transfusion-related acute lung injury. J. Clin. Invest. 122, 2661-2671.

Cech, P., Papathanassiou, A., Boreux, G., Roth, P., and Miescher, P. A.

Future translational areas of research involve the application of NETotic products as prognostic biomarkers for inflammatory disorders (e.g., sepsis, autoimmunity). For example, in patients with sepsis, the degree of NETosis may correlate with a better outcome due to enhanced bacterial clearance or a worse outcome reflecting inflammation-induced organ injury. Assays of NETotic markers (e.g., MPO-DNA complexes, NE) from cell-free fluid (e.g., plasma) may provide a quantitative measure of NETosis from archived human samples that could be correlated with clinical outcome in a variety of diseases. This knowledge may pave the way to therapeutic approaches to target NETs, such as use of histone neutralizing antibody or DNase1.

\section{ACKNOWLEDGMENT}

This work was supported by grants from the National Institutes of Health: AI079253 (to Brahm H. Segal).

(1979a). Hereditary myeloperoxidase deficiency. Blood 53, 403-411.

Cech, P., Stalder, H., Widmann, J. J., Rohner, A., and Miescher, P. A. (1979b). Leukocyte myeloperoxidase deficiency and diabetes mellitus associated with Candida albicans liver abscess. Am. J. Med. 66, 149-153.

Clark, S. R., Ma, A. C., Tavener, S. A., McDonald, B., Goodarzi, Z., Kelly, M. M., et al. (2007). Platelet TLR4 activates neutrophil extracellular traps to ensnare bacteria in septic blood. Nat. Med. 13, 463-469.

Coxon, A., Rieu, P., Barkalow, F. J., Askari, S., Sharpe, A. H., von Andrian, U. H., etal. (1996). A novel role for the beta 2 integrin CD11b/CD18 in neutrophil apoptosis: a homeostatic mechanism in inflammation. Immunity 5, 653-666.

Coxon, A., Tang, T., and Mayadas, T. N. (1999). Cytokine-activated endothelial cells delay neutrophil apoptosis in vitro and in vivo. A role for granulocyte/macrophage colonystimulating factor. J. Exp. Med. 190, 923-934.

Croker, B. A., O’Donnell, J. A., Nowell, C. J., Metcalf, D., Dewson, G., Campbell, K. J., et al. (2011). Fas-mediated neutrophil apoptosis is accelerated by Bid, Bak, and Bax and inhibited by Bcl-2 and Mcl-1. Proc. Natl. Acad. Sci. U.S.A. 108, 13135-13140.

Davidson, B. A., Vethanayagam, R. R., Grimm, M. J., Mullan, B. A., Raghavendran, K., Blackwell, T. S., etal. (2013). NADPH oxidase and Nrf2 regulate gastric aspirationinduced inflammation and acute lung injury. J. Immunol. 190, 17141724.

Eilers, B., Mayer-Scholl, A., Walker, T., Tang, C., Weinrauch, Y., and Zychlinsky, A. (2010). Neutrophil antimicrobial proteins enhance Shigella flexneri adhesion and invasion. Cell. Microbiol. 12, 1134-1143.

Farley, K., Stolley, J. M., Zhao, P., Cooley, J., and Remold-O'Donnell, E. (2012). A serpinB1 regulatory mechanism is essential for restricting neutrophil extracellular trap generation. J. Immunol. 189, 4574-4581.

Feldmann, J., Callebaut, I., Raposo, G., Certain, S., Bacq, D., Dumont, C., et al. (2003). Munc13-4 is essential for cytolytic granules fusion and is mutated in a form of familial hemophagocytic lymphohistiocytosis (FHL3). Cell 115, 461-473.

Frasch, S. C., Fernandez-Boyanapalli, R. F., Berry, K. Z., Leslie, C. C., Bonventre, J. V., Murphy, R. C., etal. (2011). Signaling via macrophage G2A enhances efferocytosis of dying neutrophils by augmentation of rac activity. J. Biol. Chem. 286, 1210812122.

Fuchs, T. A., Abed, U., Goosmann, C., Hurwitz, R., Schulze, I., Wahn, V., et al. (2007). Novel cell death program leads to neutrophil extracellular traps. J. Cell Biol. 176, 231-241.

Fuchs, T. A., Bhandari, A. A., and Wagner, D. D. (2011). Histones induce rapid and profound thrombocytopenia in mice. Blood 118, 37083714.

Fuchs, T. A., Brill, A., Duerschmied, D., Schatzberg, D., Monestier, M., Myers, D. D. Jr., etal. (2010). Extracellular DNA traps promote thrombosis. Proc. Natl. Acad. Sci. U.S.A. 107, 15880-15885.

Gantner, B. N., Simmons, R. M., Canavera, S. J., Akira, S., and Underhill, D. M. (2003). Collaborative induction of inflammatory responses by dectin- 1 and Toll-like receptor 2 . J. Exp. Med. 197, 1107-1117. 
Gao, X. P., Standiford, T. J., Rahman, A., Newstead, M., Holland, S. M., et al. (2002). Role of NADPH oxidase in the mechanism of lung neutrophil sequestration and microvessel injury induced by Gram-negative sepsis: studies in p47phox $-1-$ and gp91 phox-/- mice. J. Immunol. 168 , 3974-3982.

Garcia-Romo, G. S., Caielli, S., Vega, B., Connolly, J., Allantaz, F., Xu, Z., et al. (2011). Netting neutrophils are major inducers of type I IFN production in pediatric systemic lupus erythematosus. Sci. Transl. Med. 3, $73 \mathrm{ra} 20$.

Garlichs, C. D., Eskafi, S., Cicha, I., Schmeisser, A., Walzog, B., Raaz, D., etal. (2004). Delay of neutrophil apoptosis in acute coronary syndromes. J. Leukoc. Biol. 75, 828-835.

Geering, B., Gurzeler, U., Federzoni, E., Kaufmann, T., and Simon, $\mathrm{H}$ U. (2011). A novel TNFR1-triggered apoptosis pathway mediated by class IA PI3Ks in neutrophils. Blood 117, 5953-5962.

Gong, D., Farley, K., White, M., Hartshorn, K. L., Benarafa, C., and Remold-O'Donnell, E. (2011). Critical role of serpinB1 in regulating inflammatory responses in pulmonary influenza infection. $J$. Infect. Dis. 204, 592-600.

Goodridge, H. S., Reyes, C. N., Becker, C. A., Katsumoto, T. R., Ma, J., Wolf, A. J., et al. (2011). Activation of the innate immune receptor Dectin1 upon formation of a 'phagocytic synapse'. Nature 472, 471-475.

Graham, D. B., Robertson, C. M., Bautista, J., Mascarenhas, F., Diacovo, M. J., Montgrain, V., et al. (2007). Neutrophil-mediated oxidative burst and host defense are controlled by a Vav-PLCgamma2 signaling axis in mice. J. Clin. Invest. 117, 3445-3452.

Guiducci, C., Tripodo, C., Gong, M., Sangaletti, S., Colombo, M. P., Coffman, R. L., et al. (2010). Autoimmune skin inflammation is dependent on plasmacytoid dendritic cell activation by nucleic acids via TLR7 and TLR9. J. Exp. Med. 207, 2931-2942.

Hahn, I., Klaus, A., Janze, A. K., Steinwede, K., Ding, N., Bohling, J., et al. (2011). Cathepsin G and neutrophil elastase play critical and nonredundant roles in lung-protective immunity against Streptococcus pneumoniae in mice. Infect. Immun. 79, 4893-4901.

Hakkim, A., Fuchs, T. A., Martinez, N. E., Hess, S., Prinz, H., Zychlinsky, A., et al. (2011). Activation of the RafMEK-ERK pathway is required for neutrophil extracellular trap formation. Nat. Chem. Biol. 7, 75-77.

Hakkim, A., Furnrohr, B. G., Amann, K., Laube, B., Abed, U. A., Brinkmann, V., et al. (2010). Impairment of neutrophil extracellular trap degradation is associated with lupus nephritis. Proc. Natl. Acad. Sci. U.S.A. 107, 9813-9818.

Hu, Y., and Pham, C. T. (2005). Dipeptidyl peptidase I regulates the development of collagen-induced arthritis. Arthritis Rheum. 52, 2553 2558.

Imai, Y., Kuba, K., Neely, G. G., Yaghubian-Malhami, R., Perkmann, T., van Loo, G., et al. (2008). Identification of oxidative stress and Toll-like receptor 4 signaling as a key pathway of acute lung injury. Cell 133, 235-249.

Jun, H. S., Lee, Y. M., Song, K. D., Mansfield, B. C., and Chou, J. Y. (2011). G-CSF improves murine G6PC3-deficient neutrophil function by modulating apoptosis and energy homeostasis. Blood 117, 3881-3892.

Klein, J. B., Buridi, A., Coxon, P. Y., Rane, M. J., Manning, T., Kettritz, R., etal. (2001). Role of extracellular signal-regulated kinase and phosphatidylinositol-3 kinase in chemoattractant and LPS delay of constitutive neutrophil apoptosis. Cell. Signal. 13, 335-343.

Kraemer, B. F., Campbell, R. A., Schwertz, H., Cody, M. J., Franks, Z. Tolley, N. D., et al. (2011). Novel antibacterial activities of beta-defensin 1 in human platelets: suppression of pathogen growth and signaling of neutrophil extracellular trap formation. PLoS Pathog. 7:e1002355. doi: 10.1371/journal.ppat.1002355

Kuhns, D. B., Alvord, W. G., Heller, T., Feld, J. J., Pike, K. M., Marciano, B. E., et al. (2010). Residual NADPH oxidase and survival in chronic granulomatous disease. N. Engl. J. Med. 363, 2600-2610.

Lande, R., Ganguly, D., Facchinetti, V., Frasca, L., Conrad, C., Gregorio, J., et al. (2011). Neutrophils activate plasmacytoid dendritic cells by releasing self-DNA-peptide complexes in systemic lupus erythematosus. Sci. Transl. Med. 3, 73ra19.

Leffler, J., Martin, M., Gullstrand, B. Tyden, H., Lood, C., Truedsson, L., et al. (2012). Neutrophil extracellular traps that are not degraded in systemic lupus erythematosus activate complement exacerbating the disease. J. Immunol. 188, 35223531.

Li, P., Li, M., Lindberg, M. R., Kennett, M. J., Xiong, N., and Wang, Y. (2010). PAD4 is essential for antibacterial innate immunity mediated by neutrophil extracellular traps. J. Exp. Med. 207, 1853-1862.

Liles, W. C., Kiener, P. A., Ledbetter, J. A., Aruffo, A., and Klebanoff, S. J. (1996). Differential expression of Fas (CD95) and Fas ligand on normal human phagocytes: implications for the regulation of apoptosis in neutrophils. $J$. Exp. Med. 184, 429-440.

Marciano, B. E., Rosenzweig, S. D., Kleiner, D. E., Anderson, V. L., Darnell, D. N., Anaya-O’Brien, S., et al. (2004). Gastrointestinal involvement in chronic granulomatous disease. Pediatrics 114, 462-468.

Martinelli, S., Urosevic, M., Daryadel, A., Oberholzer, P. A., Baumann, C., Fey, M. F., et al. (2004). Induction of genes mediating interferon-dependent extracellular trap formation during neutrophil differentiation. J. Biol. Chem. 279, 44123-44132.

Massberg, S., Grahl, L., von Bruehl, M. L., Manukyan, D., Pfeiler, S., Goosmann, C., et al. (2010). Reciprocal coupling of coagulation and innate immunity via neutrophil serine proteases. Nat. Med. 16, 887-896.

McDonald, B., Urrutia, R., Yipp, B. G. Jenne, C. N., and Kubes, P. (2012). Intravascular neutrophil extracellular traps capture bacteria from the bloodstream during sepsis. Cell Host Microbe 12, 324-333.

McInturff, A. M., Cody, M. J., Elliott, E. A., Glenn, J. W., Rowley, J. W., Rondina, M. T., et al. (2012). Mammalian target of rapamycin regulates neutrophil extracellular trap formation via induction of hypoxia-inducible factor 1 alpha. Blood 120, 3118-3125.

Metzler, K. D., Fuchs, T. A., Nauseef, W. M., Reumaux, D., Roesler, J. Schulze, I., et al. (2011). Myeloperoxidase is required for neutrophil extracellular trap formation: implications for innate immunity. Blood 117, 953-959.

Meyer-Hoffert, U., and Wiedow, O. (2010). Neutrophil serine proteases: mediators of innate immune responses. Curr. Opin. Hematol. doi: 10.1097/MOH.0b013e32834115d1 [Epub ahead of print].

Mocsai, A., Zhou, M., Meng, F., Tybulewicz, V. L., and Lowell, C. A. (2002). Syk is required for integrin signaling in neutrophils. Immunity $16,547-558$

Monfregola, J., Johnson, J. L., Meijler, M. M., Napolitano, G., and Catz, S. D. (2012). MUNC13-4 protein regulates the oxidative response and is essential for phagosomal maturation and bacterial killing in neutrophils. $J$. Biol. Chem. 287, 44603-44618.
Narasaraju, T., Yang, E., Samy, R. P., Ng, H. H., Poh, W. P., Liew, A. A., et al. (2011). Excessive neutrophils and neutrophil extracellular traps contribute to acute lung injury of influenza pneumonitis. Am. J. Pathol. 179, 199-210.

Nauseef, W. M. (2012). Editorial: Nyet to NETs? A pause for healthy skepticism. J. Leukoc. Biol. 91, 353-355.

Neeli, I., Dwivedi, N., Khan, S., and Radic, M. (2009). Regulation of extracellular chromatin release from neutrophils. J. Innate Immun. 1 , 194-201.

Papayannopoulos, V., Metzler, K. D., Hakkim, A., and Zychlinsky, A. (2010). Neutrophil elastase and myeloperoxidase regulate the formation of neutrophil extracellular traps. J. Cell Biol. 191, 677-691.

Pham, C. T., Ivanovich, J. L., Raptis, S. Z., Zehnbauer, B., and Ley, T. J. (2004). Papillon-Lefevre syndrome: correlating the molecular, cellular, and clinical consequences of cathepsin C/dipeptidyl peptidase I deficiency in humans. J. Immunol. 173, 7277-7281.

Phillipson, M., and Kubes, P. (2011). The neutrophil in vascular inflammation. Nat. Med. 17, 1381-1390.

Pillay, J., den Braber, I., Vrisekoop, N., Kwast, L. M., de Boer, R. J., Borghans, J. A., et al. (2010). In vivo labeling with ${ }^{2} \mathrm{H}_{2} \mathrm{O}$ reveals a human neutrophil lifespan of 5.4 days. Blood 116 , 625-627.

Pitt, J., and Bernheimer, H. P. (1974). Role of peroxide in phagocytic killing of pneumococci. Infect. Immun. 9, $48-52$.

Raptis, S. Z., Shapiro, S. D., Simmons, P. M., Cheng, A. M., and Pham, C. T. (2005). Serine protease cathepsin $G$ regulates adhesion-dependent neutrophil effector functions by modulating integrin clustering. Immunity 22, 679-691.

Reeves, E. P., Lu, H., Jacobs, H. L., Messina, C. G., Bolsover, S., Gabella, G., et al. (2002). Killing activity of neutrophils is mediated through activation of proteases by K+ flux. Nature 416, 291-297.

Remijsen, Q., Vanden Berghe, T., Wirawan, E., Asselbergh, B., Parthoens, E., De Rycke, R., et al. (2011). Neutrophil extracellular trap cell death requires both autophagy and superoxide generation. Cell Res. 21, 290-304.

Renshaw, S. A., Timmons, S. J., Eaton, V., Usher, L. R., Akil, M., Bingle, C. D., et al. (2000). Inflammatory neutrophils retain susceptibility to apoptosis mediated via the Fas death receptor. J. Leukoc. Biol. 67, 662-668. 
Saffarzadeh, M., Juenemann, C., Queisser, M. A., Lochnit, G., Barreto, G., Galuska, S. P., et al. (2012). Neutrophil extracellular traps directly induce epithelial and endothelial cell death: a predominant role of histones. PLOS ONE 7:e32366. doi: 10.1371/journal.pone.0032366

Saitoh, T., Komano, J., Saitoh, Y., Misawa, T., Takahama, M., Kozaki, T., et al. (2012). Neutrophil extracellular traps mediate a host defense response to human immunodeficiency virus-1. Cell Host Microbe 12, 109-116.

Sato, K., Kadiiska, M. B., Ghio, A. J., Corbett, Y. J., Fann, C., Holland, S. M., et al. (2002). In vivo lipid-derived free radical formation by NADPH oxidase in acute lung injury induced by lipopolysaccharide: a model of ARDS. FASEB J. 16, 1713-1720.

Savill, J. S., Wyllie, A. H., Henson, J. E., Walport, M. J., Henson, P. M., and Haslett, C. (1989). Macrophage phagocytosis of aging neutrophils in inflammation. Programmed cell death in the neutrophil leads to its recognition by macrophages. J. Clin. Invest. 83, 865-875.

Segal, B. H., Davidson, B. A., Hutson, A. D., Russo, T. A., Holm, B. A., Mullan, B., et al. (2007). Acid aspiration-induced lung inflammation and injury are exacerbated in NADPH oxidase-deficient mice. Am. J. Physiol. Lung Cell. Mol. Physiol. 292, L760-L768.

Segal, B. H., Veys, P., Malech, H., and Cowan, M. J. (2011). Chronic granulomatous disease: lessons from a rare disorder. Biol. Blood Marrow Transplant. 17(Suppl. 1), S123-S131.

Shohet, S. B., Pitt, J., Baehner, R. L., and Poplack, D. G. (1974). Lipid peroxidation in the killing of phagocytized pneumococci. Infect. Immun. 10, 1321-1328.

Sica, A., and Mantovani, A. (2012). Macrophage plasticity and polarization: in vivo veritas. J. Clin. Invest. $122,787-795$.
Soehnlein, O., Kai-Larsen, Y., Frithiof, R., Sorensen, O. E., Kenne, E., Scharffetter-Kochanek, K., et al. (2008a). Neutrophil primary granule proteins HBP and HNP1-3 boost bacterial phagocytosis by human and murine macrophages. J. Clin. Invest. 118, 3491-3502.

Soehnlein, O., Zernecke, A., Eriksson, E. E., Rothfuchs, A. G., Pham C. T., Herwald, H., et al. (2008b). Neutrophil secretion products pave the way for inflammatory monocytes. Blood 112, 1461-1471.

Soehnlein, O., Weber, C., and Lindbom, L. (2009a). Neutrophil granule proteins tune monocytic cell function. Trends Immunol. 30, 538-546.

Soehnlein, O., Zernecke, A., and Weber, C. (2009b). Neutrophils launch monocyte extravasation by release of granule proteins. Thromb. Haemost. 102, 198-205.

Standish, A. J., and Weiser, J. N. (2009). Human neutrophils kill Streptococcus pneumoniae via serine proteases. J. Immunol. 183, 2602-2609.

Stark, M. A., Huo, Y., Burcin, T. L., Morris, M. A., Olson, T. S., and Ley, K. (2005). Phagocytosis of apoptotic neutrophils regulates granulopoiesis via IL-23 and IL-17. Immunity 22, 285-294.

Thomas, G. M., Carbo, C., Curtis, B. R., Martinod, K., Mazo, I. B., Schatzberg, D., et al. (2012). Extracellular DNA traps are associated with the pathogenesis of TRALI in humans and mice. Blood 119, 6335-6343.

Urban, C. F., Ermert, D., Schmid, M., Abu-Abed, U., Goosmann, C., Nacken, W., etal. (2009). Neutrophil extracellular traps contain calprotectin, a cytosolic protein complex involved in host defense against Candida albicans. PLoS Pathog. 5:e1000639. doi: 10.1371/journal.ppat.1000639

van den Berg, J. M., van Koppen, E., Ahlin, A., Belohradsky, B. H., Bernatowska, E., Corbeel, L., et al.
(2009). Chronic granulomatous disease: the European experience. PLoS ONE 4:e5234. doi: 10.1371/journal.pone.0005234

Vethanayagam, R. R., Almyroudis, N G., Grimm, M. J., Lewandowski, D. C., Pham, C. T., Blackwell, C. T., et al. (2011). Role of NADPH oxidase versus neutrophil proteases in antimicrobial host defense. PLoS ONE 6:e28149. doi: 10.1371/journal.pone.0028149

Villanueva, E., Yalavarthi, S., Berthier, C. C., Hodgin, J. B., Khandpur, R., Lin, A. M., etal. (2011). Netting neutrophils induce endothelial damage, infiltrate tissues, and expose immunostimulatory molecules in systemic lupus erythematosus. J. Immunol. 187, 538-552.

Wang, Y., Li, M., Stadler, S., Correll, S., Li, P., Wang, D., et al. (2009). Histone hypercitrullination mediates chromatin decondensation and neutrophil extracellular trap formation. J. Cell Biol. 184, 205-213.

Weinrauch, Y., Drujan, D., Shapiro, S. D., Weiss, J., and Zychlinsky, A (2002). Neutrophil elastase targets virulence factors of enterobacteria. Nature 417, 91-94.

Winkelstein, J. A., Marino, M. C., Johnston, R. B. Jr., Boyle, J., Curnutte, J., Gallin, J. I., et al. (2000). Chronic granulomatous disease: report on a national registry of 368 patients. Medicine (Baltimore) 79, 155-169.

Yamada, M., Gomez, J. C., Chugh, P. E., Lowell, C. A., Dinauer, M. C., Dittmer, D. P., et al. (2011). Interferon-gamma production by neutrophils during bacterial pneumonia in mice. Am. J. Respir. Crit. Care Med. 183, 1391-1401.

Yamashita, K., Takahashi, A., Kobayashi, S., Hirata, H., Mesner, P. W., Kaufmann, S. H., et al. (1999). Caspases mediate tumor necrosis factor-alphainduced neutrophil apoptosis and downregulation of reactive oxygen production. Blood 93, 674-685.
Yipp, B. G., Petri, B., Salina, D., Jenne, C. N., Scott, B. N., Zbytnuik, L. D., et al. (2012). Infection-induced NETosis is a dynamic process involving neutrophil multitasking in vivo. Nat. Med. 18, 1386-1393.

Yousefi, S., Mihalache, C., Kozlowski, E., Schmid, I., and Simon, H. U. (2009). Viable neutrophils release mitochondrial DNA to form neutrophil extracellular traps. Cell Death Differ. 16, 1438-1444.

Zhang, Q., Raoof, M., Chen, Y., Sumi, Y., Sursal, T., Junger, W., et al. (2010). Circulating mitochondrial DAMPs cause inflammatory responses to injury. Nature 464, 104-107.

Conflict of Interest Statement: The authors declare that the research was conducted in the absence of any commercial or financial relationships that could be construed as a potential conflict of interest.

Received: 18 December 2012; accepted: 07 February 2013; published online: 01 March 2013.

Citation: Almyroudis NG, Grimm MJ, Davidson BA, Röhm M, Urban CF and Segal BH (2013) NETosis and NADPH oxidase: at the intersection of host defense, inflammation, and injury. Front. Immunol. 4:45. doi: 10.3389/ fimmu.2013.00045

This article was submitted to Frontiers in Molecular Innate Immunity, a specialty of Frontiers in Immunology.

Copyright (c) 2013 Almyroudis, Grimm, Davidson, Röhm, Urban and Segal. This is an open-access article distributed under the terms of the Creative Commons Attribution License, which permits use, distribution and reproduction in other forums, provided the original authors and source are credited and subject to any copyright notices concerning any thirdparty graphics etc. 